\title{
MUSIFICATION OF SEISMIC DATA
}

\author{
Ryan McGee \\ Media Arts and Technology \\ University of California, Santa Barbara \\ ryanemat.ucsb. edu
}

\begin{abstract}
Seismic events are physical vibrations induced in the earth's crust which follow the general wave equation, making seismic data naturally conducive to audification. Simply increasing the playback rates of seismic recordings and rescaling the amplitude values to match those of digital audio samples (straight audification) can produce eerily realistic door slamming and explosion sounds. While others have produced a plethora of such audifications for international seismic events (i.e. earthquakes), the resulting sounds, while distinct to the trained auditory scientist, often lack enough variety to produce multiple instrumental timbres for the creation of engaging music for the public. This paper discusses approaches of sonification processing towards eventual musification of seismic data, beginning with straight audification and resulting in several musical compositions and new-media installations containing a variety of seismically derived timbres.
\end{abstract}

\section{INTRODUCTION}

Supported by the Australia Council for the Arts Music Board, the original goal of our research was to accentuate sonic differences in the audification of individual seismic events around the world whilst searching for musical qualities within the sounds. Work began during a residency at the AlloSphere Research Center at the University of California, Santa Barbara - a unique venue for multi-channel sound spatialization ${ }^{1}$ In addition to a highly spatialized, multi-channel work for the AlloSphere, two pieces of stereo, electronica-style music were produced that could be used to engage the public and have since received over 45,000 plays on SoundCloud. These electronica pieces, coupled with a new live-streaming seismic sound engine have been used in long-term public installations (Section 47).

Starting by replicating previous seismic audification techniques[1] [2] [9] using MATLAB, our sound processing grew to include a number of granular and frequency-domain effects to obtain a greater variety of sound timbres produced from a single seismic recording. We then used extremes of granular processing and frequency-domain filtering to accentuate sonic differences between separate seismic events as well as separate recordings of the same event.

1 http://allosphere.ucsb.edu/ Commercial 4.0 International License. The full terms of the License are available at http://creativecommons.org/licenses/by-nc/4.0

\section{David Rogers}

\author{
College of Fine Arts \\ University of New South Wales \\ dvreallshookup.org
}

\section{AUDIFICATION OF SEISMIC DATA}

Since seismic recordings are a form of physical data[3] obeying the general wave equation, the process of making them audible is simply a matter of rescaling seismometer data recordings to the range of digital audio samples, $[-1.0,1.0]$, and playing them at rates fast enough enter our range of hearing $(20 \mathrm{~Hz}-20 \mathrm{kHz})$. The typical range for seismic waves is $0.1-3 \mathrm{~Hz}$, so increasing the playback rate by a factor of $100-1000 \mathrm{X}$ is common.

Seismometers record activity 3-dimensionally along vertical, East-West, and North-South axes as shown in Figure 11. Seismic recording stations categorize their recordings by sample rate, gain sensitivity, and orientation. For instance a channel code of $\mathbf{B H Z}$ would indicate a broad band, high gain, vertically (z-axis) orientated recording. Broad band channels are indicative of a $10 \mathrm{~Hz}-$ $80 \mathrm{~Hz}$ sampling rate (specified in the header of each recording), which are desirable for audification as they are the highest available sample rates for any event. Likewise, high gain channels are desirable to produce more amplitude resolution in the resulting sounds. Differences amongst the audification of separate axes are subtle (Figure 2), so the vertical, Z, channel is typically used by default, but it is possible to synchronize and mix the audifications of all 3 orientations together to produce a slightly fuller sound.

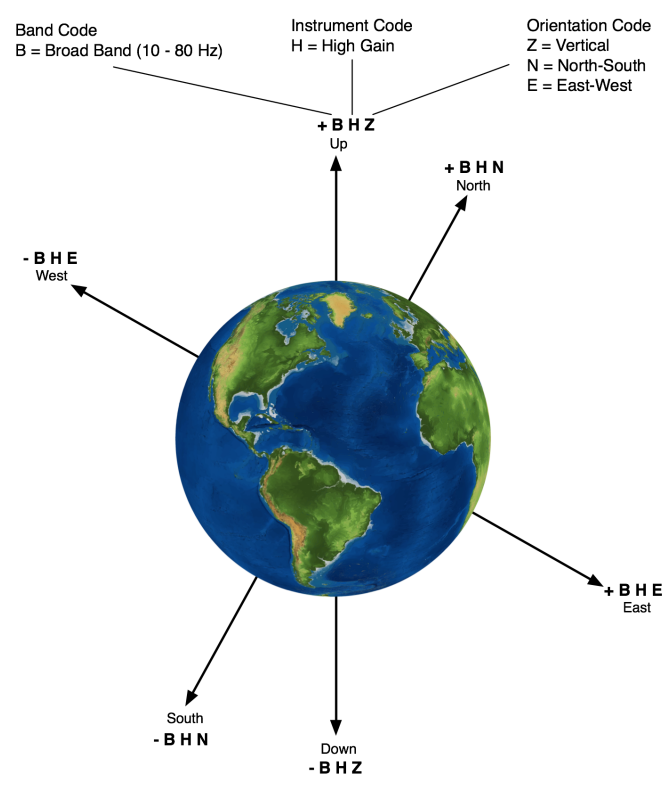

Figure 1: Orientation of Seismic Recording Channels 
Our experiments began with data collected from the February 21st, 2011 magnitude 6.1 Christchurch, New Zealand Earthquake. IRIS (Incorporated Research Institutions for Seismology|: provides an online interface for accessing a database of current and past seismic events around the world ${ }^{3}$ Figure 2 shows the original waveform produced from audification at 276 times the original speed of the Christchurch event followed by examples of the sound processing techniques described in the following sections.

\section{SEISMIC SONIFICATION TECHNIQUES}

Wanting to explore more sonic variation for each seismic event without straying from the data, we devised several means of audio processing without using parameter mapping[4] so that the seismic data sets would remain as the only sound generators. This is an important distinction within the field of sonification since most techniques involve the mapping of data to parameters of subjectively chosen sound generators. With this work, the original seismic waveforms are accelerated and scaled to generate sound, and variety is achieved by resampling, filtering, granulation, timestretching, and pitch shifting. Since the definition of audification limits processing to resampling, scaling, and minor filtering [3] we consider any further modifications to the sound as entering the realm of sonification. The following processes were used heavily in the creation of the Christchurch and Haiti compositions (Section 4 .

Several sound examples to accompany the following sections are online at http://i-e-i.wikispaces.com/ Auditory+Display

\subsection{Synchronous Granulation}

Granulation of sound is the process of slicing a sound into several sound "grains" creating segments lasting 1 to 100 milliseconds[5]. If the grains are played back in order then the original sound results. One can repeat each adjacent grain a number of times to result in a new sound 5 times longer than the original. Choosing an arbitrary duration for each repeated grain will result in several discontinuities in the sound. For example, a grain's amplitude may start at 0.23 and end on -0.72 . When repeated, this jump in amplitude would produce an undesirable click in the sound. To solve this a short amplitude window (envelope or ramp) is applied to each grain so each always starts and ends at amplitude 0 . Next, the grains are overlapped so there is less audible beating from the windowing. In this work, synchronous granulation refers to methods of granulation that involve several grains of identical duration and windowing. Audified earthquakes can be characterized by an initial high frequency, high amplitude sound that decays over time like hitting a snare drum. Synchronous granulation has the effect of time-stretching these sounds, repeatedly emphasizing each grain, which emphasizes the unique decay of each earthquake. However, a drawback is that the amplitude windowing can produce additional low-frequency beating artifacts in the sound.

\subsection{Asynchronous Granulation Based on Zero-Crossings}

Asynchronous granulation implies that each sound grain will have have different characteristics. In our case, the duration of each grain varies over time based on an algorithm that chooses the start

\footnotetext{
$\sqrt[2]{\text { http://www.iris.edu }}$

3 http://ds.iris.edu/wilber3/find_event
}

and end points for each grain based on the location of zero crossings within the sound, which are points where the wave's amplitude is equal to 0 . Zero crossings may occur often, sometimes even less than $1 \mathrm{~ms}$ apart, so a minimum duration for each grain is also specified. The convenience of using zero crossings is that windowing is not needed since the grains will already start and end on 0 . Another quality of zero crossings is that they usually indicate the beginning of an impulse or large transient within the sound. When asynchronous grains are repeated multiple times, the transient, impulsive portions of each earthquake are emphasized, creating stuttering rhythms unique for each event.

\subsection{Time-Stretching, Pitch Shifting, and Filtering via Phase Vocoding}

The phase vocoder[6] is a complex process used for spectral analysis and resynthesis, allowing for frequency-domain filtering. Its process breaks a sound into multiple segments of equal duration and uses a Fast Fourier Transform to analyze the frequency spectrum of each segment. One may interpolate multiple spectra between two segments to extend the duration of a sound while maintaining its frequency content. If one time-stretches a sound in such a fashion and then alters the playback rate, the result becomes a change in pitch without a change in duration (unlike audification). The spectra of each segment can also be manipulated to apply filtering effects. Removing all frequencies below a certain amplitude threshold has the effect of de-noising a sound, leaving only the most prominent frequencies. This de-noising can be taken to extremes to leave only a few partials in each sound, ultimately producing unique tones and chords for seismic events.

\section{SEISMIC COMPOSITIONS AND INSTALLATIONS}

\subsection{Christchurch (2012)}

https://soundcloud.com/seismicsounds/ christchurch-earthquake

Christchurch uses a single seismic recording from the nearest station to the February 21st, 2011 Christchurch earthquake. The piece begins with a build-up of several reversed audifications of the event, time-stretched at different speeds. Then, the strong impact from the raw audification is heard, followed immediately by a chaotic granulated version emphasizing the loudest points in the impact. A tone fades in that is an extremely time-streched, pitch shifted version of the event with all but the most dominant partials filtered out of the sound. Other versions of this tone eventually overlap at manually coordinated harmonic pitch intervals. The event is played back using several different time-strech factors and synchronous granulations during the course of the piece. Timing becomes more ordered and apparent until rhythmic granulations and tones lead to a final build-up, ending with another raw audification. The result is an exploration of timbral variety from a single seismic recording as the only sound generator.

\subsection{Haiti (2012)}

https://soundcloud.com/seismicsounds/

\section{haiti-earthquake-12th-january}

Haiti uses seismic recordings of the 12th January, 2010 magnitude 7.0 Haiti event and explores variety of sounds produced from 



Figure 2: Audification Processing: De-Noising and Asynchronous Granulation

the same event recorded by the nearest 12 stations. The piece begins with granulated audifications of each station played in succession from the furthest to nearest station at the same playback rate. A brief recording of sensor noise from each station is played along with the granulations. Since each station has its own distinct sound, these noises represent signatures of each station as the listener moves nearer to the quake. A slow melody plays in the background that was generated by filtering out all but the most prominent single frequency from the spectra of each noise signature. A rhythmic sound in the background was generated from the impact of each station played back at high-speed in succession again from furthest to nearest. As this rhythm gradually increases in intensity a lower noise grows in the background, which is a time stretched recording of the impact played in reverse to further emphasize the backwards (far to near) build-up of the piece. At the climax the impact from the nearest station plays, followed by impacts from the other stations - this time increasing in distance. In the background the low growl of the time-stretched recording fades away. The piece becomes chaotic after the main event using asynchronous granulation based on zero-crossings.

\subsection{Shadow Zone Shadows (2012)}

https://soundcloud.com/seismicsounds/ shadow-zone-shadows

Shadow Zone Shadows was an abstract sound study that spatialized seismic audifications according to the geographic location of their impact and simulated their spatial traversal through the earth. This piece was presented in a dark space with no visuals within the AlloSphere, allowing the listener to imagine being placed at the center of the earth while experiencing a series of earthquakes occurring and moving around them over a 3D loudspeaker array. A variety of international seismic events were used ranging in magnitude from 5.5 (Los Angeles) to 9.1 (Sumatra). The piece used custom spatialization software that allowed the programming of spatial sound trajectories synchronized with specific points in the seismic data (start of event and reflections). Just as the seismic data is accelerated to become an audible audification, the spatial trajectories used were simulated at speeds much faster than seismic waves actually propagate through the earth.

The AlloSphere uses a spherical 54.1 channel loudspeaker configuration consisting of 3 rings of speakers (lower, ear-level, and upper). For the sound spatialization we mapped the latitude and longitude of seismic events to virtual sound source points on the surface of the loudspeaker sphere. A distance-based amplitude panning [7] algorithm was used with custom $\mathrm{C}++$ software to pan sounds between loudspeaker locations.

\subsection{DOMUS (2014-15)}

http: / / domus.urbanaction.org

DOMUS was an experimental architecture installation incorporating spatialized seismic sound and light within a hexayurt (Figure 3). 6 mid range speakers, 4 subwoofers, and a 360 degree LED pixel sphere chandelier displayed live seismic audifications and the Christchurch and Haiti compositions continuously from $10 \mathrm{am}$ to $10 \mathrm{pm}$ for the 7-month period of installation (October 2014-May 2015) at Materials and Applications, Los Angeles ${ }^{4}$

4 http://www.emanate.org/past-exhibitions/domus 
The low-frequency seismic compositions resonated throughout the two story hexayurt DOMUS structure, emphasizing their seismic nature while demonstrating an "architecture of life" - a biophilic design model that responds and reflects the natural world [8]. The light chandelier by Rene Christiar 5 visualized the audifications by mapping sound frequency and amplitude to light hue and brightness respectively.
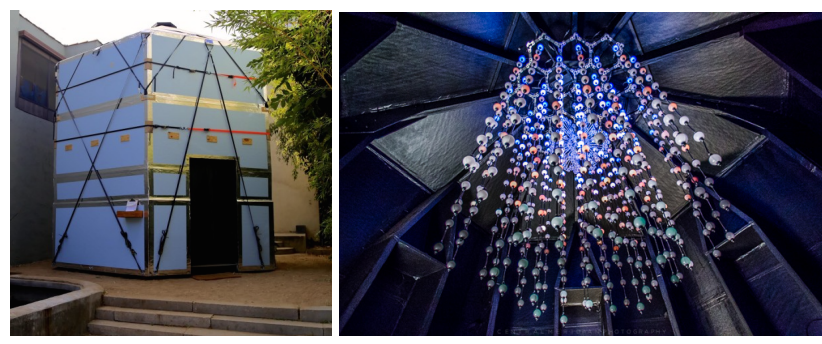

Figure 3: DOMUS Seismic Sound and Light Architecture

\subsection{Sounds of Seismic (2012-16)}

Sounds of Seismic (SOS $]^{6}$ auditory display streaming system broadcasts continuous seismic sound generated from realtime collected global earthquake data. Influenced by John Cage's Variations VII (1966), SOS is a "Musique Concrete" like audio composition in which the score is algorithmically generated by seismic waveform data. SOS can also be used as a listening tool for earth scientists to listen to a specific sensor on the Global Seismic Network (GSN). The conceptual framework of SOS is to create greater social awareness of natural ecological systems by generating multi-channel seismic sound electronica creating an infinite computational earth system soundscape.

SOS is an ongoing long term project built on our C++ Earthquake Sound Engine (ESE) and custom Python seismic data acquisition scripts by Stock Plum. Real-time miniSEED data is collected from Incorporated Research Institutions for Seismology (IRIS) and piped through ESE using the techniques outlined in Sections 2 and 3 We seek collaboration with public or private institutions providing streaming audio services and digital legacy design to present this infinite computational seismic audio sound performance.

\section{SUMMARY}

Through the desire to find musicality within seismic data we have discovered that time-domain granulation and frequency-domain filtering techniques are especially useful for deriving timbral variety between otherwise similar seismic audifications. In particular, a de-noising filter which removes all frequencies below a variable amplitude threshold is useful to produce unique tones and chords for seismic events. Asynchronous granulation based on zero crossings emphasizes transients (impacts) and produces unique stuttering rhythms for events. While the phase vocoder and synchronous granulation provide other means to time-stretch sounds, the required windowing and spectral interpolation will depart further

\footnotetext{
${ }^{5}$ http://renechristen.net

http://www.sos.allshookup.org
}

from the original sound characteristics in comparison with granulation based on zero crossings.

While many combinations of the aforementioned sound processing techniques are possible, we emphasize that the seismic data is ultimately the only source of sound generation. Because seismic data is physical wave data, using time-stretching and pitchshifting allows us to magnify and focus on qualities already present in the data without mapping to arbitrary sound generators. We consider the ability to produce multiple timbres from a single data set without parameter mapping crucial to exploring the variety of musicality naturally present within seismic events. We have sought to create enough timbral variety necessary to produce every "instrumental" part of engaging pieces of electronic music via processing rather than mapping, so seismic data remains as the original, sole sound generator.

The ultimate goal of this research is to create a generative, dynamic audification-based musak which can highlight resilience and awareness of the natural world in which we inhabit. For inspiration we have looked back to the work of Haywards[1] and Dombois[9] and look forward to creating a music that is both a meaningful tool for geophysics monitoring as well as an engaging means of raising public seismic awareness. Ongoing work with SOS (Section 4.5) provides a platform for endless, live seismic data accompanied by real-time sound processing and, eventually, music generation.

\section{ACKNOWLEDGMENTS}

The authors would like to thank the Australian Council for the Arts Music Board and AlloSphere Research Group for supporting this research.

\section{REFERENCES}

[1] C. Hayward, "Listening to the earth sing," in Auditory Display: Sonification, Audification, and Auditory Interfaces. Addison-Wesley, 1994.

[2] S. Speeth, "Seismometer sounds," in Journal of the Acoustical Society of America, vol. 33, 1961, pp. 909-916.

[3] F. Dombois and G. Eckel, Audification, T. Hermann, A. Hunt, and J. G. Neuhoff, Eds. Berlin, Germany: Logos Verlag, 2011.

[4] F. Grond and J. . Berger, Parameter Mapping Sonification, T. Hermann, A. Hunt, and J. G. Neuhoff, Eds. Berlin, Germany: Logos Verlag, 2011.

[5] C. Roads, "Granular synthesis," in Microsound. MIT Press, 2001, ch. 3.

[6] _ - "Spectrum analysis: The phase vocoder," in The Computer Music Tutorial. MIT Press, 1996, ch. 13, pp. 566-577.

[7] T. Lossius, P. Baltazar, and T. de La Hogue, "DBAP-DistanceBased Amplitude Panning," in Proceedings of 2009 International Computer Music Conference, Montreal, Canada, no. 1, 2009.

[8] M. M. Stephen R Kellert, Judith Heerwagen, Biophilic Design : The Theory, Science, and Practice of Bringing Buildings to Life. Wiley, 2008.

[9] F. Dombois, "Using audification in planetary seismology," in Proceedings of the International Conference on Auditory Display, 2001. 